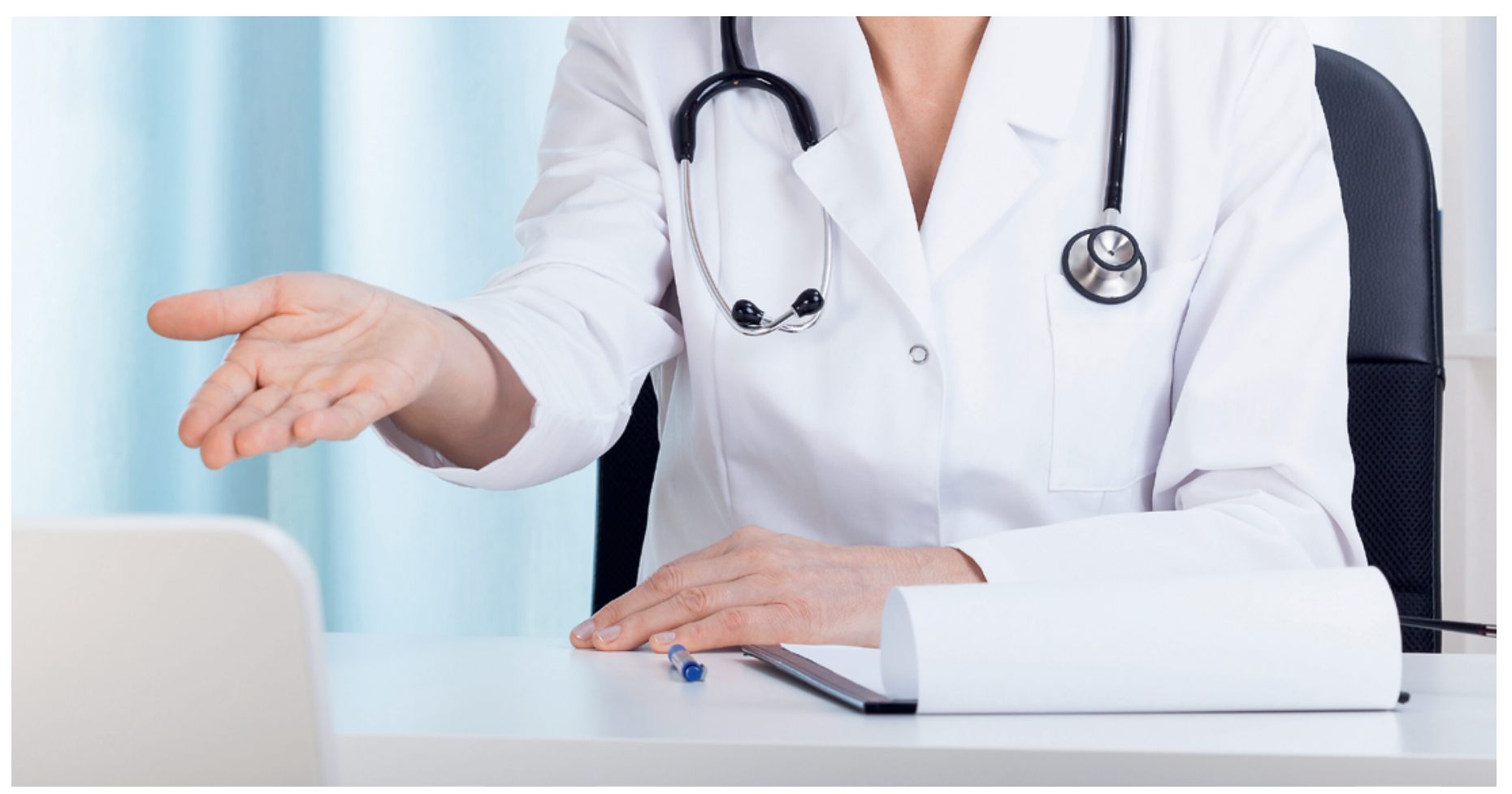

\title{
Disharmonie in Dysbalance
}

\section{Enrico Danieli}

Dr. med., Facharzt für Allgemeine Innere Medizin, Mitglied FMH

Der befehlende Blick in Richtung Stuhl, auf dem ich noch sitze, um die Fragen der Ärztin/des Arztes zu beantworten, dieser Blick spricht zu mir; er meint, so will mir scheinen, körperliche Untersuchung. Da, an diesem Punkt im Raum, habe ich mich auszuziehen, gegenüber der am Tisch sitzenden Doktorin/des Dok-

\section{Tempoverlust durch Verweilen in Kabinen ist nicht mehr einzukalkulieren.}

tors. Ja, ich bin ein wenig überempfindlich, ein wenig gehemmt in diesen Dingen, mit denen man zeitgemässer umgehen könnte. Und sinnloserweise und der Situation unangepasst erinnere ich mich, einmal gehört zu haben, dass Ärztinnen/ Ärzte den Patienten (mich?) während der Entkleidung beobachten, um erste Eindrücke zu gewinnen von dessen in einem Vorprimärstadium schon präsenten kognitiv-koordinatorisch inkohärenten Unzulänglichkeiten, verwertbaren Red Flags beginnender Hirnfunktionsalterationen. Ist dies der Grund, weshalb die schützenden
Vorhänge der einstigen Kabinen durch inexistente Glaswände moderner Vitrinen ersetzt werden? Oder ist das öffentliche Sich-der-Kleider-Entledigen ein Zeichen der Zeit: alles transparent, Privates verpönt, keine Eigenheit? Aber: Der Schutz durch Kabinen verhalf Scham und Schüchternheit zu kaschieren. Doch Tempoverlust durch Verweilen in Kabinen ist nicht mehr einzukalkulieren. Man nahm sich in vorhanggeschützten Orten in stickiger Luft ein wenig Zeit, um zu sinnieren, der eigenen Körperlichkeit nachzustudieren.

Jetzt alles auf dem Stuhl. Dem Sitzstuhl. Dem Redestuhl. Dem Entkleidungsstuhl. Dem Stuhl der Verurteilung. Vor der Ärztin/dem Arzt mich der Kleider zu entledi-

\section{All mein Elend steigert sich. Die Knopflöcher sind zu klein für die Knöpfe.}

gen ist ein Kunststück mit hohem Schwierigkeitsgrad. Was zuerst, was danach? Solche Fragen bedrängen, blockieren mich. Er ist ungeschickt, hat deutliche Koordi- 
nationsstörungen, er weiss nicht, wie sich entkleiden, hat kein Entkleidungsziel, zeigt Zeichen der Dysmetrie. Die Knöpfe des Hemdes. Fatal. All mein Elend steigert sich. Die Knopflöcher sind zu klein für die Knöpfe. Ich reisse. Knöpfe weg. Kein Kommentar. Ein Blick durch mich hindurch. Aus Verärgerung wegen des Zeitverlusts klopfen Fingerbeeren auf Tischkanten. Triumph der Bestätigung des Dys-Verdachtes? Die Strümpfe. Alles so eng. Ich schwanke, kann mich nicht abstützen. Deutliche Dyskinesien, einfach zu erkennen. Einbeinstand: Da ist er, dieser Test, rôle signature: Wenn positiv, alles positiv, alles verloren. Ich schaffe diese Übung nicht, nicht gleich. Das Elend vor dem Ende. Und erst das Unterhemd, definitiver Schuldspruch. Ich bin nassgeschwitzt, weil das Einfachste nicht gelingt, weil ich mich erbärmlich, zutiefst entwürdigt fühle. Das Unterhemd klebt, klebt fest, ist verleimt. Wie soll ich es über den Kopf ziehen? Es rollt sich, zieht sich zusammen,

\section{Der Höhepunkt der Demütigung steht bevor.}

Jetzt, in diesem Moment, denke ich heute, hätte ich aufgeben sollen.

ein dickes Seil. Ziehen, stossen, nichts dehnt sich. Das Gegenüber ohne Regung, stumm. Ein einziger Satz könnte meine Verzweiflung lindern, mich erretten: «Ach ja, das ist ekelhaft, ich kenne das!» Irgendwas. Doch nun, den Strick über den Augen, höre ich, dass der Slip noch wartet. Wartet? Wer wartet? Noch? Und auf was? Der Höhepunkt der Demütigung steht bevor. Jetzt, in diesem Moment, denke ich heute, hätte ich aufgeben sollen. Einfach weggehen. Fort. Unangekleidet aus der Praxis fliehen, ich kann mich nicht ankleiden, ich bin verklebt, doch draussen werde ich aufgegriffen, zurückgebracht, weggeschafft. Aber nun, Stück für Stück entkleidet, beurteilt, entwertet, gibt es kein Zurück. Befindlichkeitswallungen Einzelner sind Luxus.
Doktorin/Doktor tut schliesslich nur das, was zu tun ist. Kopf, Hals, Herz, Lunge. Haut. $9 / 10$ nackt auf dem Stuhl, auf den Kleidern sitzend. Ich schwitze, ich siede, ich dampfe. Ich hinterlasse eine Schweissspur. Rieche ich? Ja, absonderlich. Nun hinauf auf den Umkleidestuhl, da hinauf. Der Stuhl bewegt sich, dreht sich. Ich krieche auf allen vieren, Vierfüssler-Gang. Noch ein Test. Da ist sie, die alles beweisende Dysbalance. Man schaut nicht, man schweigt. Ich richte mich auf, stehe da, um schwankendes Gleichgewicht bemüht, auf der Bergspitze. Füsse, Beine. Und dann: 1/10. «Das Slip weg!» Ich schüttle den Kopf, doch man sieht mich nicht von dort unten in der Talsohle. Wiederholung, ungehalten: «Das Slip!» «Nein», rufe ich laut, «nein, nicht das! Den!» Man schaut mir zum ersten Mal in die Augen. «Jetzt Slip weg!» «Pils?», frage ich. Eine Dummheit, das Wort rückwärts zu sagen, ich weiss. «Den!», wird gerufen. «Nein», flüstere ich, «das Pils!» Die diagnostizierten Dysthymismen, Dyslogismen, Dyslalismen summieren die Dys, sie zwingen zur Teilnahme an gruppenspezifischer teamdynamischer frühtherapeutischer DysDysMentia-Prophylaxe. Das ist alles. Schluss. Ende der Geschichte. Den DysDysMentia-Kurstherapiebeginn für Erstsymptomträger habe ich verpasst, auch den Reminder, den Reminder des Reminders. In beschwerlichen Situationen hilft rückwärts oder verkehrtherum gehen oder stehen: Mein windiges Verhalten fängt die Instabilität meiner Lage mit Leichtigkeit auf. Pils? Slip! Oder umgekehrt. «... manchmal war es ihm unangenehm, dass er nicht auf dem Kopf gehen konnte ...» (Georg Büchners Lenz). Das ist es: Mit Hilfe des Auf-dem-Kopf-Gehens (-Denkens, -Sprechens) gelingt es, zu widerstehen!

Bildnachweis

@ Katarzyna Bialasiewicz | Dreamstime.com (Symbolbild) 